\title{
Coupled dipole method with an exact long-wavelength limit and improved accuracy at finite frequencies
}

\author{
Adel Rahmani \\ Atomic Physics Division, National Institute of Standards and Technology, Gaithersburg, Maryland 20899-8423 \\ Patrick C. Chaumet \\ Institut Fresnel (Unité Mixte de Recherche 6133), Faculté des Sciences et Techniques de St. Jérôme, \\ Avenue Escadrille Normandie-Niemen, F-13397 Marseille Cedex 20, France \\ Garnett W. Bryant \\ Atomic Physics Division, National Institute of Standards and Technology, Gaithersburg, Maryland 20899-8423
}

Received September 6, 2002

\begin{abstract}
We present a new formulation of the coupled dipole method that accounts for local-field effects and is exact in the long-wavelength limit. This formulation also leads to improved accuracy of the description of lightscattering processes at finite frequencies. (C) 2002 Optical Society of America

OCIS codes: $260.2110,290.0290$.
\end{abstract}

Since its introduction in the early seventies ${ }^{1}$ the discrete dipole approximation, also called the coupled dipole method (CDM), has been used extensively to study many problems, such as light scattering by arbitrary objects, ${ }^{2-4}$ near-field molecular probes, ${ }^{5}$ optical forces, ${ }^{6}$ and nanosources in microcavities. ${ }^{7}$ The versatility of the method resides in the simplicity of its formalism. An arbitrary scatterer can always be viewed as a collection of polarizable subunits. Provided that the sizes of the subunits are small enough compared with the local spatial variations of the field, the dipole approximation applies, and each subunit can be represented by a polarizability $\alpha$. Several formulations of the CDM exist. They differ by the polarizabilities used to describe the optical response of the subunits. Nevertheless, the polarizabilities always reduce to the Clausius-Mossotti (CM) expression in the long-wavelength limit. As it is often pointed out, for a uniform excitation the CM expression for the polarizability of a small particle is exact in the long-wavelength limit. However, as demonstrated by Draine, ${ }^{2}$ when the CM polarizability is used within the CDM to describe a small particle as a collection of dipoles, one does not get the exact result in the long-wavelength limit. The reason for this is that the common assumption that all dipoles must have the same optical response, irrespective of their environment, is flawed. Indeed, near interfaces, one must account for the particular local environment of each dipole.

In this Letter we present a form of the CDM that is exact in the long-wavelength regime. This form is achieved by derivation of a self-consistent local-field correction that accounts for the particular environment of each subunit. Accordingly, the performance of the CDM at finite frequencies is improved as well. We start with a general derivation of the self-consistent local-field correction and of the corresponding polarizability. Consider an arbitrary scatterer discretized over a cubic lattice with spacing $d$. The selfconsistent local field at subunit $i$ is given by

$$
\mathbf{E}_{i}^{\text {loc }}=\mathbf{E}_{i}^{0}+\sum_{j \neq i} \mathrm{~F}_{i j} \alpha_{j} \mathbf{E}_{j}^{\text {loc }}
$$

where $\mathbf{E}_{i}{ }^{0}$ is the incident field at subunit $i$ and $\mathrm{F}$ is the free-space field susceptibility, ${ }^{8}$ i.e., $F_{i j} \alpha_{j} \mathbf{E}_{j}{ }^{\text {loc }}$, gives the electric field at location $i$ produced by subunit $j$. The sum over $j$ runs over all the subunits $(j \neq i)$ that form the scatterer. Our aim is to find the exact local-field factor in the long-wavelength limit (electrostatic approximation) from which we will infer the polarizability. After making the electrostatic approximation and introducing macroscopic field $\mathbf{E}^{m}$, which is related to the incident field by $\mathbf{E}^{m}\left[1+\mathrm{L}_{i}\left(\epsilon_{i}-\right.\right.$ 1) $/(4 \pi)]=\mathbf{E}_{i}{ }^{0}$, with depolarization tensor $L_{i}$, we find that Eq. (1) becomes

$$
\mathbf{E}_{i}^{\mathrm{loc}}=\left(1+\mathrm{L}_{i} \frac{\epsilon_{i}-1}{4 \pi}\right) \mathbf{E}_{i}^{m}+\sum_{j \neq i} \mathrm{~F}_{i j} \alpha_{j} \mathbf{E}_{j}^{\mathrm{loc}}
$$

where 1 is the identity tensor and $\epsilon_{i}$, assumed to be scalar for simplicity, is the permittivity of subunit $i$. By definition, local-field tensor $\Lambda_{i}$ satisfies

$$
\mathbf{E}_{i}^{\text {loc }}=\Lambda_{i} \mathbf{E}_{i}^{m} .
$$

Using the fact that the polarization can be written as

$$
\begin{aligned}
\mathbf{P}_{i} & =\frac{\alpha_{i}}{d^{3}} \mathbf{E}_{i}^{\text {loc }}, \\
& =\frac{\epsilon_{i}-1}{4 \pi} \mathbf{E}_{i}^{m}, \\
& =\frac{\epsilon_{i}-1}{4 \pi} \Lambda_{i}^{-1} \mathbf{E}_{i}^{\text {loc }},
\end{aligned}
$$


we can express the polarizability in terms of the local-field tensor:

$$
\alpha_{i}=\frac{\epsilon_{i}-1}{4 \pi} \Lambda_{i}^{-1} d^{3} .
$$

We derive the local-field tensor by use of Eqs. (3) - (7) to express the local field in terms of the macroscopic field in Eq. (2), leading to

$$
\Lambda_{i}=\mathrm{C}_{i}+\sum_{j \neq i} \mathrm{~F}_{i j} \frac{\epsilon_{j}-1}{4 \pi} d^{3} \mathrm{C}_{j}{ }^{-1} \mathrm{C}_{i},
$$

where the tensors $\mathrm{C}_{i}$ are defined as

$$
\mathrm{C}_{i}=1+\frac{\epsilon_{i}-1}{4 \pi} \mathrm{L}_{i}
$$

The depolarization tensor, $\mathrm{L}_{i}$, is essentially a geometrical factor whose value is determined by the shape of the object. $^{9}$ Note that to arrive at Eq. (8) we assumed that the initial, static field $\mathbf{E}^{0}$ was uniform (static limit of a plane wave, for instance). If this were not the case, there would simply be an additional term in the sum on the right-hand side of Eq. (8) to account for the variation of $\mathbf{E}^{0}$ from one subunit to another.

The polarizability of Eq. (7) reduces to the CM expression only for a single, isolated dipolar scatterer or a subunit lying away from any interface. In the general case the polarizability accounts for the environment of the subunit via the local-field tensor [Eq. (8)]. Note that because of its dependence on the environment the polarizability can be anisotropic even for an isotropic material (scalar $\epsilon$ ). We emphasize that Eq. (7) replaces the CM expression. This means that the usual radiation reaction correction ${ }^{2}$ can now be applied to Eq. (7). All the results presented below include the radiation-reaction correction.

To illustrate the method presented above, we consider a slab (infinite along directions $x$ and $y$ ) of material with permittivity $\epsilon$ and thickness $t$ along direction $z$. This configuration has an analytic solution that will be our reference. Note that for a slab (as well as for ellipsoids and infinite cylinders ${ }^{10}$ ) $L_{i}$ is uniform over the slab. For more-complex geometries $L_{i}$ is not constant and can easily be calculated numerically. ${ }^{9}$ ) We emphasize that although the geometry of a slab allows a two-dimensional treatment we describe the slab as a three-dimensional object, using an extension of the CDM to periodic structures. ${ }^{11}$ This approach allows us to use three-dimensional expressions for the polarizabilities and will make it easier to apply the present approach to more-complex scatterers. The case of a slab makes the physical content of Eq. (7) obvious. A subunit near the surface of the slab will respond (be polarized) differently depending on the polarization of the incident field, because of the subunit's particular environment near the interface. However, a subunit that lies within the bulk of the slab will be have an isotropic response.

We focus on the behavior of the new formulation of the CDM at finite frequencies. Indeed, in the long-wavelength limit, Eq. (7) is exact by design. We first consider a dielectric slab $(\epsilon=20, t=50 \mathrm{~nm})$ illuminated by a plane wave, with wavelength $\lambda=400 \mathrm{~nm}$ and angle of incidence $\theta=50^{\circ}$, polarized in the $x z$ plane. The slab occupies the region $0 \leq z \leq 50 \mathrm{~nm}$ and is discretized into 20 layers. We choose a large value of $\epsilon$ to emphasize the effects of the new formulation of the CDM. We compare in Fig. 1 the $x$ component of the macroscopic field outside and inside the slab, for the old (conventional) and new forms of the CDM, with the exact result. The old CDM predicts strong, nonphysical oscillations of the field inside the slab. These oscillations have been pointed out before, although they have not been explained. ${ }^{12-14}$ They result from the erroneous assumption that the bulk local-field correction can be used for every subunit. Indeed, the assumption that each subunit of the CDM is well described by the CM polarizability and experiences a local-field correction $(\epsilon+2) / 3$ is incorrect near interfaces. However, the new form of the CDM reduces drastically the oscillations of the macroscopic field inside the slab. As a result, the reflected and the transmitted fields are improved as well. We emphasize that the new local-field correction is most important at interfaces. In general, the new polarizability differs significantly from the CM value only for the first two or three layers near an interface.

To illustrate further the influence of the local-field correction on the calculation of the scattered field, we present in Figs. 2 and 3 the relative error (in percent) of the transmitted field as a function of the number of layers used to discretize the slab. The incident light is polarized in the $x z$ plane or along $y$, and we represent the $x$ or the $y$ components of the field. The $z$ component has a behavior similar to the $x$ component. The inclusion of local-field corrections [Eq. (7)] improves significantly the convergence of the CDM for dielectric (Fig. 2), metals (Fig. 3), and strongly absorbing media (not shown). Because the optical response of each subunit accounts for its local environment, the induced dipole moment at each subunit is calculated more precisely,

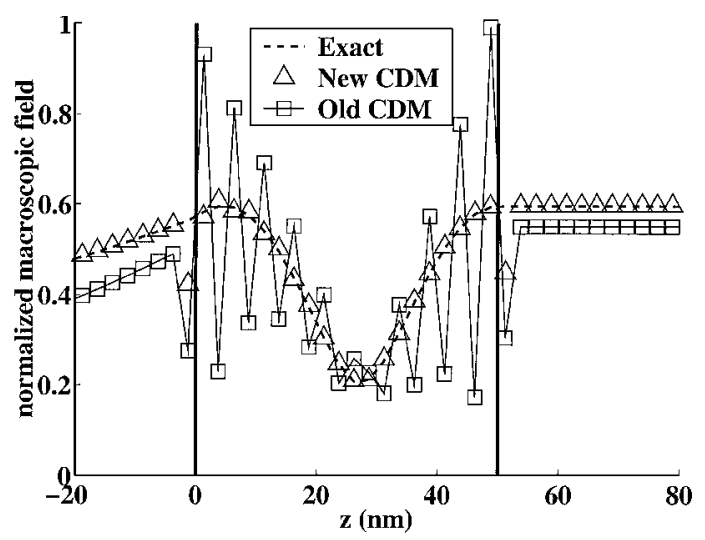

Fig. 1. Macroscopic field outside and inside a dielectric slab. The slab occupies the region $0 \leq z \leq 50 \mathrm{~nm}$ delimited by the vertical lines. The plane wave $(\lambda=400 \mathrm{~nm})$ is incident from the left of the figure. Angle $\theta=50^{\circ}$, permittivity of the slab $\epsilon=20$ (see text for details). 


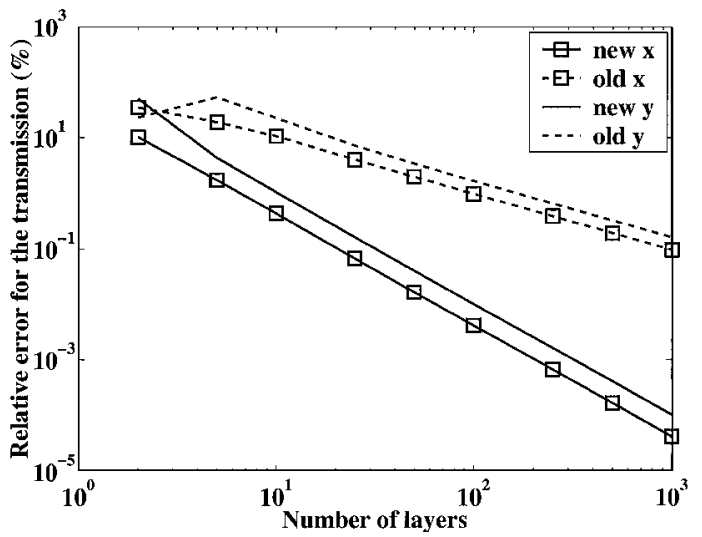

Fig. 2. Relative error of the field transmitted through a slab. Results for the $x$ and the $y$ components are plotted as a function of the number of discretization layers. The parameters of the calculation are $\epsilon=20, t=50 \mathrm{~nm}, \theta=$ $50^{\circ}$, and $\lambda=600 \mathrm{~nm}$.

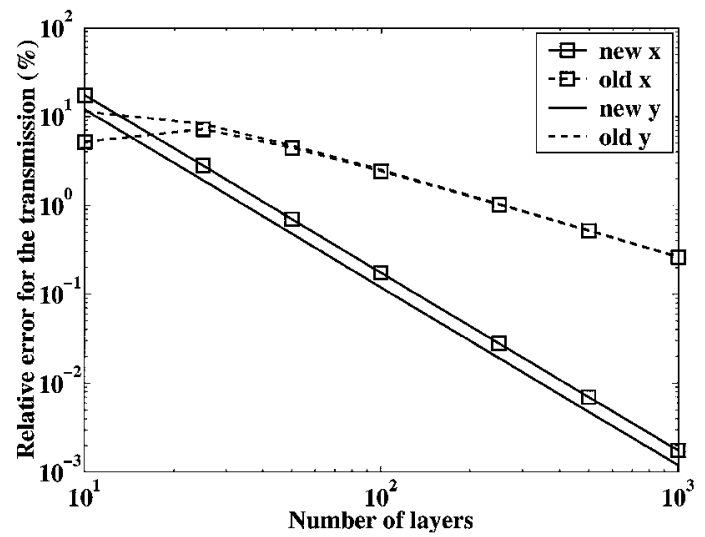

Fig. 3. Same as Fig. 2 but for an aluminum slab and parameters $\epsilon=-34.5+8.5 i, t=50 \mathrm{~nm}, \theta=50^{\circ}$, and $\lambda=$ $488 \mathrm{~nm}$.

and therefore the error on the scattered field is reduced.

In summary, we have presented a new form of the $\mathrm{CDM}$ in which the local field is derived so that the
CDM is exact in the long-wavelength limit. The polarizability of the subunits is expressed in terms of a local-field tensor that is derived self-consistently and accounts for the local environment of each subunit. Accordingly, the convergence of the CDM at finite frequencies is drastically improved, and fewer subunits are needed for a given accuracy. In this Letter we explicitly considered a slab to make the physical basis for the new form of the CDM clear. It will be interesting to consider more-complex geometries. Indeed, calculations for spherical scatterers show that the performance of the CDM for three-dimensional scatterers can be significantly improved.

A. Rahmani's e-mail address is adel.rahmani@ ec-lyon.fr.

\section{References}

1. E. M. Purcell and C. R. Pennypacker, Astrophys. J. 186, 705 (1973).

2. B. T. Draine, Astrophys. J. 333, 848 (1988).

3. B. T. Draine and J. Goodman, Astrophys. J. 405, 685 (1993).

4. B. T. Draine and P. J. Flatau, J. Opt. Soc. Am. A 11, 1491 (1994), and references therein.

5. A. Rahmani, P. C. Chaumet, and F. de Fornel, Phys. Rev. A 63, 023819 (2001).

6. P. C. Chaumet, A. Rahmani, and M. Nieto-Vesperinas, Phys. Rev. Lett. 88, 123601 (2002).

7. A. Rahmani and G. W. Bryant, Phys. Rev. A 65, 033817 (2002).

8. J. D. Jackson, Classical Electrodynamics, 2nd ed. (Wiley, New York, 1980).

9. A. D. Yaghjian, Proc. IEEE 68, 248 (1980).

10. J. A. Stratton, Electromagnetic Theory (McGraw-Hill, New York, 1941).

11. P. C. Chaumet, A. Rahmani, and G. W. Bryant have prepared a manuscript called "Generalization of the coupled dipole method to periodic structures."

12. N. Kar and A. Bagchi, Solid State Commun. 31, 645 (1980).

13. W. L. Mochán and R. G. Barrera, Phys. Rev. Lett. 55, 1192 (1985).

14. G. P. M. Poppe and C. M. J. Wijers, Physica B 167, 221 (1990). 